\title{
Observation of a Complex Nanoscale Magnetic Structure in a Hexagonal Fe Monolayer
}

\author{
K. von Bergmann, ${ }^{1, *}$ S. Heinze, ${ }^{1}$ M. Bode, ${ }^{1}$ E. Y. Vedmedenko, ${ }^{1}$ G. Bihlmayer, ${ }^{2}$ S. Blügel,${ }^{2}$ and R. Wiesendanger ${ }^{1}$ \\ ${ }^{1}$ Institute of Applied Physics, University of Hamburg, Jungiusstraße 11, 20355 Hamburg, Germany \\ ${ }^{2}$ Institut für Festkörperforschung, Forschungszentrum Jülich, 52425 Jülich, Germany
}

(Received 2 December 2005; published 26 April 2006)

\begin{abstract}
We have observed a novel magnetic structure in the pseudomorphic Fe monolayer on $\operatorname{Ir}(111)$. Using spin-polarized scanning tunneling microscopy we find a nanometer-sized two-dimensional magnetic unit cell. A collinear magnetic structure is proposed consisting of $15 \mathrm{Fe}$ atoms per unit cell with 7 magnetic moments pointing in one and 8 moments in the opposite direction. First-principles calculations verify that such an unusual magnetic state is indeed lower in energy than all solutions of the classical Heisenberg model. We demonstrate that the complex magnetic structure is induced by the strong Fe-Ir hybridization.
\end{abstract}

DOI: 10.1103/PhysRevLett.96.167203

A focus in recent research has been put on the magnetic properties of thin films. Investigations have demonstrated that the interplay between symmetry, nearest-neighbor distance, and hybridization with the substrate plays a crucial role for the magnetic ground state. Up to now only two different magnetic ground states of homoatomic monolayers (ML) have been found experimentally: while the ferromagnetic (FM) state is accessible by various surface sensitive techniques it is much more challenging to prove antiferromagnetic (AFM) order such as the $c(2 \times 2)$-AFM state $[1,2]$. However, theoretical calculations have predicted a variety of much more complex magnetic structures of homoatomic MLs. A model system to induce complex magnetic order is an AFM material on a triangular lattice: this leads to frustration of the AFM interactions and can be realized by a hexagonal ML on a (111) fcc substrate [3-6]. In such systems noncollinear magnetic structures with up to 4 atoms per unit cell have been predicted. If stabilized in the fcc structure even a prototypical FM element such as Fe has been reported to show a noncollinear magnetic structure with a continuous rotation of the magnetic moments resulting in a spin spiral $[7,8]$. Numerous experimental and theoretical studies have also been performed for thin $\mathrm{Fe}$ films on $\mathrm{Cu}(001)$ and $\mathrm{Cu}(111)$ proposing $\mathrm{AFM}$ sequences normal to the surface but FM within each layer [9-12]. Apparently, the fcc environment induces complex magnetic structures in Fe even in thin films.

This Letter reveals novel magnetic behavior found for the pseudomorphic hexagonal Fe ML on $\operatorname{Ir}(111)$. Spinpolarized scanning tunneling microscopy (SP-STM) measurements indicate a complex nanometer-scale magnetic structure consisting of $15 \mathrm{Fe}$ atoms per unit cell. Our firstprinciples calculations show that the proposed magnetic state is indeed more favorable than all possible magnetic solutions describable by the classical Heisenberg model, which includes interactions between neighbors of arbitrary distance. We find that the $3 d-5 d$ hybridization between the Fe ML and the Ir substrate plays a key role for the unusual magnetic properties.
PACS numbers: 75.70.Ak, 68.37.Ef, 71.15.Mb, 75.25.+z

The experiments were performed in a multichamber ultrahigh vacuum system with separate chambers for substrate and tip preparation, metal deposition, and low temperature STM measurements [13]. The $\operatorname{Ir}(111)$ single crystal surface was prepared by Ar-ion etching at room temperature and subsequent annealing $(T=1350 \mathrm{~K})$. Fe was evaporated from a rod and deposited after the substrate reached room temperature. All STM measurements were performed with tip and sample held at $T=13 \mathrm{~K}$, and a magnetic field up to strength of $B= \pm 2.5 \mathrm{~T}$ could be applied normal to the sample surface, i.e., along the tip axis.

An overview of a typical sample of 0.6 atomic layers (AL) of Fe on the stepped $\operatorname{Ir}(111)$ surface is shown in Fig. 1. The topography in Fig. 1(a) shows that in addition to the first AL already small areas of the second AL are present. While the first AL appears flat with edges running mostly along the main crystallographic directions of the substrate the second AL shows an unordered reconstruction. The simultaneously measured map of differential conductance $(d I / d U)$ in Fig. 1(b) reveals two different types of ML areas. They can be distinguished by their electronic structure and the direction of the edges, which is characteristic for pseudomorphic areas and ML with a stacking fault [14]. Atomic resolution of the $\operatorname{Ir}(111)$ substrate and the Fe ML attached to a step within one image

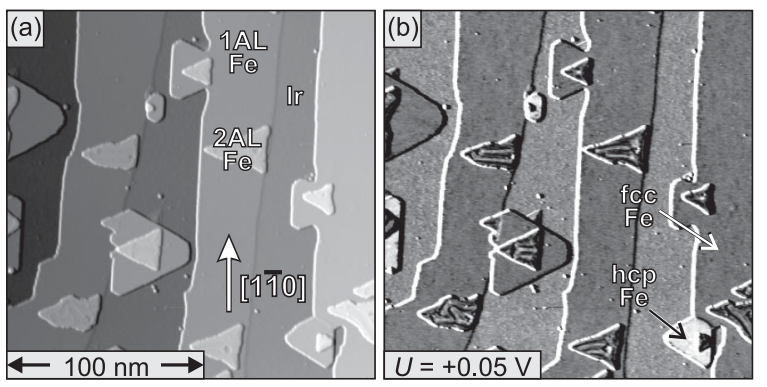

FIG. 1. STM images of $0.6 \mathrm{AL}$ of Fe on $\operatorname{Ir}(111)$ : (a) topography and (b) simultaneously acquired $d I / d U$ map. 
shows undistorted hexagonal order for both surfaces (not shown) and the nearest-neighbor distance is in good agreement with the expected Ir value of $a=2.715 \AA$. Assuming that all protrusions mark atom positions we conclude that the ML areas attached to the Ir steps continue the Ir atom arrangement and thus refer to them as fcc in the following.

While measurements with a bare tungsten tip reflect the topographic structure of a sample, in SP-STM images also the magnetic structure is observed $[1,15]$. Figures 2(a)2(c) show SP-STM images of the same surface area of the fcc Fe ML measured with an Fe-coated tip at different applied magnetic field strength $B$. Typically Fe-coated tips are magnetized perpendicular to the tip axis, i.e., sensitive to the magnetization in the sample surface-plane, but due to the application of an external magnetic field, the magnetization of the tip can be turned towards its axis which results in a sensitivity to the out-of-plane component of the sample magnetization [2]. The constant-current image measured at $B=0 \mathrm{~T}$ [Fig. 2(a)] appears featureless, while with applied magnetic field a superstructure is found which maintains its size and shape but increases in amplitude

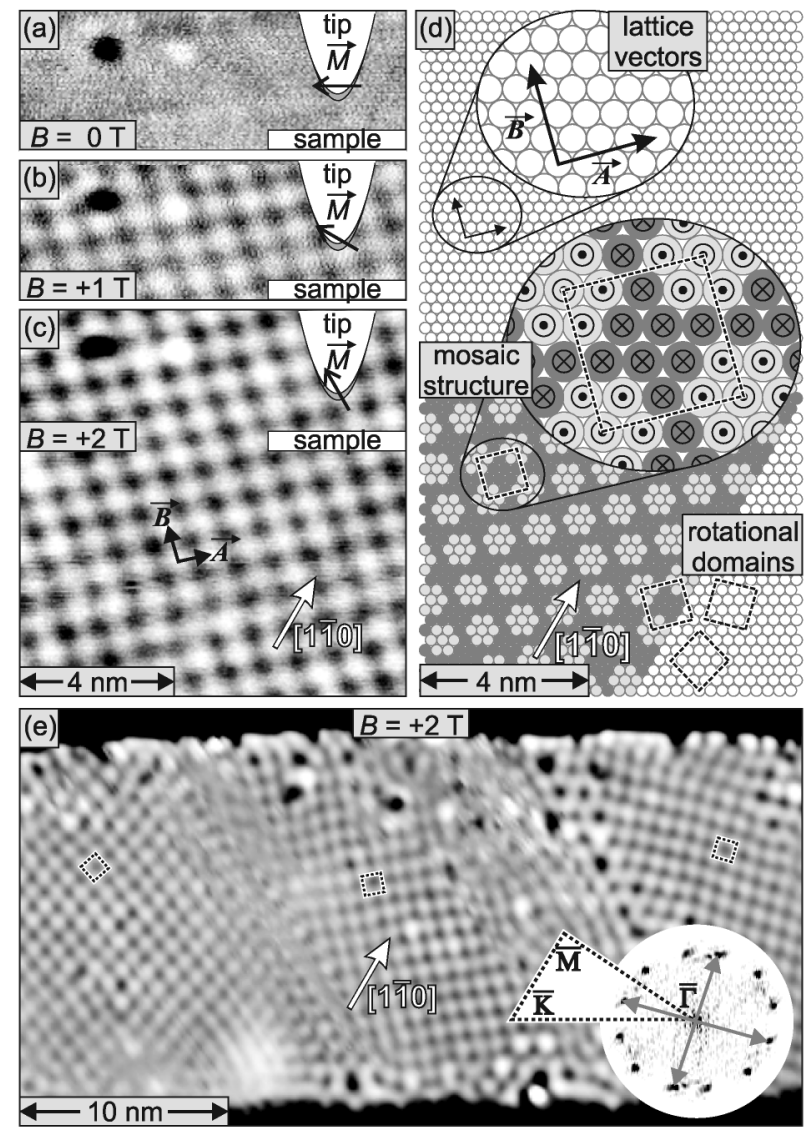

FIG. 2. Magnetism of the fcc Fe ML on $\operatorname{Ir}(111)$. (a)-(c) SPSTM images of the same area measured with an Fe-coated tip at $B$ as indicated $(U=+0.05 \mathrm{~V}, I=0.5 \mathrm{nA}) ;(\mathrm{d})$ structure model on the basis of the experimental data; (e) STM image measured with an Fe-coated tip displaying all possible rotational domains $(U=+0.05 \mathrm{~V}, I=0.25 \mathrm{nA})$, inset: FT of the image and a sketch of the I-2D-BZ. from $B=+1 \mathrm{~T}$ [Fig. 2(b)] to $B=+2 \mathrm{~T}$ [Fig. 2(c)]. Considering the impact of $B$ on the tip's magnetization (see insets), we conclude that the observed superstructure is of magnetic origin with an out-of-plane magnetization direction of the fcc Fe ML. Since in zero magnetic field the superstructure was also occasionally observed with out-ofplane sensitive [GdFe- or Cr-coated [15]] tips, we can exclude a formation of the superstructure as a result of the applied magnetic field. For hcp Fe ML islands no magnetic superstructure was observed.

The magnetic unit cell of the fcc Fe ML observed in Figs. 2(b) and 2(c) is approximately square with one diagonal along a close packed row of the $\operatorname{Ir}(111)$ substrate and lattice vectors $\mathbf{A}$ and $\mathbf{B}$ with a length of $\approx 1 \mathrm{~nm}$. A symmetric height distribution suggests that bright and dark areas are equivalent except for opposite out-of-plane magnetization components. A structure model based on these experimental findings is shown in Fig. 2(d): assuming a magnetic structure commensurate with the atomic lattice, only the displayed connections $\mathbf{A}$ and $\mathbf{B}$ between the atoms are possible within the experimental error. The resulting magnetic unit cell has lattice vectors $|\mathbf{A}|=|\mathbf{B}|=\sqrt{13} a=$ $0.979 \mathrm{~nm}$, the angles are $-46.1^{\circ}$ and $+46.1^{\circ}$ with respect to the [1 $1 \overline{1} 0$ ] direction and it consists of 15 atoms. Assuming for simplicity collinearity and the same magnetic moment for all Fe atoms there must be a small spin imbalance, which is minimized by the arrangement sketched in the middle of Fig. 2(d) where 7 magnetic moments point in one direction and 8 moments in the opposite one. For this magnetic structure, which will be referred to as $(7: 8)$ mosaic structure in the following, a lot of domains shifted by one atomic site are possible. Because of the sixfold symmetry of the hexagonal lattice it also has three rotational domains, all of which can be observed in the STM image in Fig. 2(e). The inset shows the Fourier transform (FT) of the image together with a sketch of the irreducible part of the two-dimensional Brillouin zone (I-2D-BZ). The three sets for the rotational domains can clearly be distinguished, one is indicated by arrows.

In order to understand the surprising magnetism of the Fe ML on $\operatorname{Ir}(111)$ we have performed first-principles calculations based on density functional theory. We apply the full-potential linearized augmented plane wave method in film geometry as implemented in the FLEUR code [16]. The surface was modeled by an asymmetric film consisting of one Fe layer in fcc stacking on four layers of $\operatorname{Ir}(111)$. We have used the generalized-gradient approximation (GGA) to the exchange-correlation functional [17]. The lattice constant obtained for fcc Ir is $3.89 \AA$ which is only $1 \%$ larger than the experimental value. The equilibrium Fe-Ir interlayer distance for the FM state is $d=2.10 \AA$. For calculations with the 2D unit cell of 15 atoms we have used 100 basis functions per atom and six $\mathbf{k}_{\|}$points in the I-2D-BZ. The spin spiral calculations have been performed with 120 basis functions per atom and $1024 \mathbf{k}_{\|}$points in the full 2D-BZ. 
First we focus on calculations for the collinear magnetic structure suggested from the experimental observations. Indeed, the total energy of the proposed (7:8) mosaic structure is found to be about $3 \mathrm{meV} / \mathrm{Fe}$ atom lower than that of the FM solution [18]. The Fe atoms in the (7:8) mosaic structure have an average absolute value of the magnetic moment of $\bar{m}_{\mathrm{Fe}}=2.96 \mu_{\mathrm{B}}$ varying about $\pm 0.06 \mu_{\mathrm{B}}$ from site to site, which supports the simple assumption of nearly constant moments. The values are slightly enhanced compared to the FM state with $m_{\mathrm{Fe}}=$ $2.92 \mu_{\mathrm{B}}$. The magnetic moments of Ir atoms change only very little and are smaller than $0.1 \mu_{\mathrm{B}}$. Although the total energy difference is quite small it supports the model proposed from the experimental study. To investigate the influence of the $3 d-5 d$ hybridization between the Fe ML and the Ir substrate, the Fe-Ir interlayer distance was reduced by only $3 \%$ (to $d=2.04 \AA$ ). Surprisingly, the energy gain of the $(7: 8)$ mosaic structure with respect to the FM state increased to $15 \mathrm{meV} / \mathrm{Fe}$ atom, demonstrating the crucial role of hybridization for the magnetic order.

In Fig. 3 we compare the experimental SP-STM data with our calculated results based on the spin-polarized extension [19] of the Tersoff-Hamann (TH) model [20]. Within the TH model the local density of states (LDOS) in the vacuum is proportional to the $d I / d U$ signal. In the spatially averaged $d I / d U$ spectrum of the fcc Fe ML, shown in Fig. 3(a), two broad peaks are observed, one at about $-0.5 \mathrm{~V}$, i.e., in the occupied sample states, and one at about $+0.5 \mathrm{~V}$ in the unoccupied states. The calculated LDOS of the FM state shows a single sharp peak in the unoccupied states, while the (7:8) mosaic structure possesses peaks at $-0.35 \mathrm{~V}$ and $+0.65 \mathrm{~V}$. Apart from a shift by about $0.15 \mathrm{~V}$ there is a good qualitative agreement between the mosaic structure and the measured spectrum. We attribute the larger intensity of the experimental data at negative bias voltage to the enhanced influence of tip states [21]. The calculated SP-STM images [19] for the (7:8) and
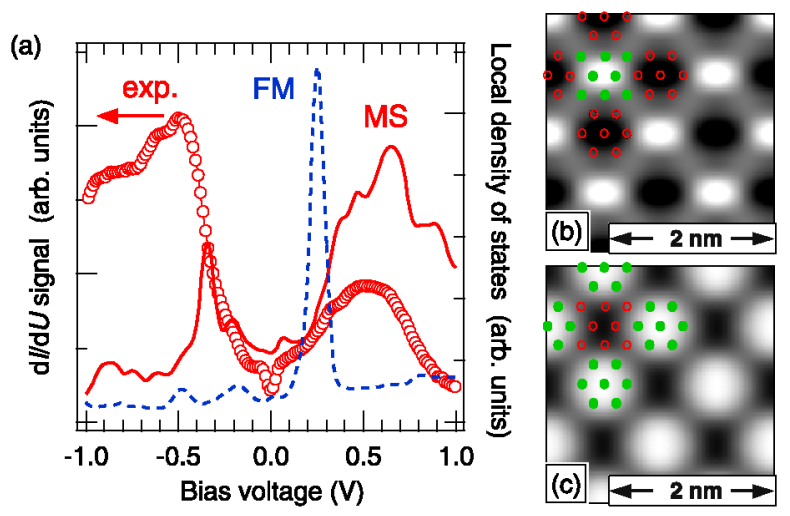

FIG. 3 (color online). (a) Experimental $d I / d U$ spectrum in comparison to the calculated vacuum LDOS of the FM and the (7:8) mosaic structure (MS). (b), (c) Calculated SP-STM images for the (7:8) and (8:7) domains of the magnetic mosaic structure $(U=+0.15 \mathrm{~V}$, tip-sample distance $7.2 \AA$, spin polarization of the tip 0.4).
(8:7) domains of the mosaic structure in Figs. 3(b) and 3(c) show that the magnetic unit cell dominates the appearance while the atomic-scale magnetic order cannot be resolved. Both images look very similar and are in excellent agreement with the experiment. Since in the measurements only one superstructure is found we expect that the domains cannot be distinguished experimentally.

So far we have considered only two collinear magnetic configurations. To include noncollinear states and to shed light onto the origin of this peculiar magnetic structure, we investigate the system within the framework of the classical Heisenberg model, $H=\sum_{i<j} J_{i j} \mathbf{S}_{i} \mathbf{S}_{j}$, where $\mathbf{S}_{i}$ corresponds to the local moment at site $i$. The electronic structure is contained in the exchange constants $J_{i j}$, whose Fourier transformed quantities, $J(\mathbf{Q})$, are determined by the total energy $E(\mathbf{Q})$ of helical spin waves as calculated from first-principles. For a given reciprocal vector $\mathbf{Q}$ of a flat spin spiral, the magnetic moment of an atom at site $\mathbf{R}$ is given by $\mathbf{m}(\mathbf{R})=m_{\mathrm{s}}(\cos (\mathbf{Q R}), \sin (\mathbf{Q R}), 0)$, where $m_{\mathrm{s}}$ is the spin moment per atom. It has been pointed out in the past [5] that the calculation of $E(\mathbf{Q})$ along the highsymmetry directions of the I-2D-BZ includes a vast number of states in the magnetic phase space. At high symmetry points of the $2 \mathrm{D}-\mathrm{BZ}$ we find well-known magnetic states: at the $\bar{\Gamma}$ point the FM state is obtained, the $\bar{M}$ point denotes the row-wise (RW)-AFM state, and the $\overline{\mathrm{K}}$ point corresponds to the Néel state $[5,6]$.

The calculated energy $E(\mathbf{Q})$ is shown in Fig. 4 for hexagonal Fe MLs on different substrates on the Ir lattice constant. The simplest configuration is a free-standing unsupported ML (UML). A global energy minimum is observed at the $\bar{\Gamma}$ point, i.e., the FM state is the ground state solution $\left(m_{\mathrm{Fe}}=3.04 \mu_{\mathrm{B}}\right)$. The energy per $\mathrm{Fe}$ atom rises very steeply with $|\mathbf{Q}|$ and the nearest-neighbor exchange constant favors ferromagnetism while constants between more distant atoms are much smaller. On a (111) metal substrate with a full $d$ shell such as Ag, the magnetic properties of the Fe ML are modified only weakly due to hybridization [6]. Near $\bar{\Gamma}$ the observed energy $E(\mathbf{Q})$ is very similar to that of the UML while the magnetic moment is reduced to $m_{\mathrm{Fe}}=2.93 \mu_{\mathrm{B}}$.

However, the situation changes drastically when Ir with its partly filled $5 d$ band is the substrate. At the equilibrium interlayer distance $(d=2.10 \AA)$, the FM state remains the global energy minimum of the spin spirals [22]. Nevertheless, the exchange constants between nearest and next nearest-neighbor atoms are greatly reduced and the energy differences to other metastable magnetic solutions have decreased significantly, e.g., for the RW-AFM state from $156 \mathrm{meV} / \mathrm{Fe}$ atom for the UML to only $41 \mathrm{meV} / \mathrm{Fe}$ atom. $E(\mathbf{Q})$ is also affected, and we find a plateaulike feature, only $10 \mathrm{meV} / \mathrm{Fe}$ atom higher than the FM state, for $|\mathbf{Q}| \approx 0.2 \times 2 \pi / a$. This plateau can be related to the experimentally observed magnetic periodicity (A and $\mathbf{B}$ correspond to $|\mathbf{G}|=0.277 \times 2 \pi / a$ in reciprocal space). Upon a small reduction of $d$ to $2.04 \AA$ and to 


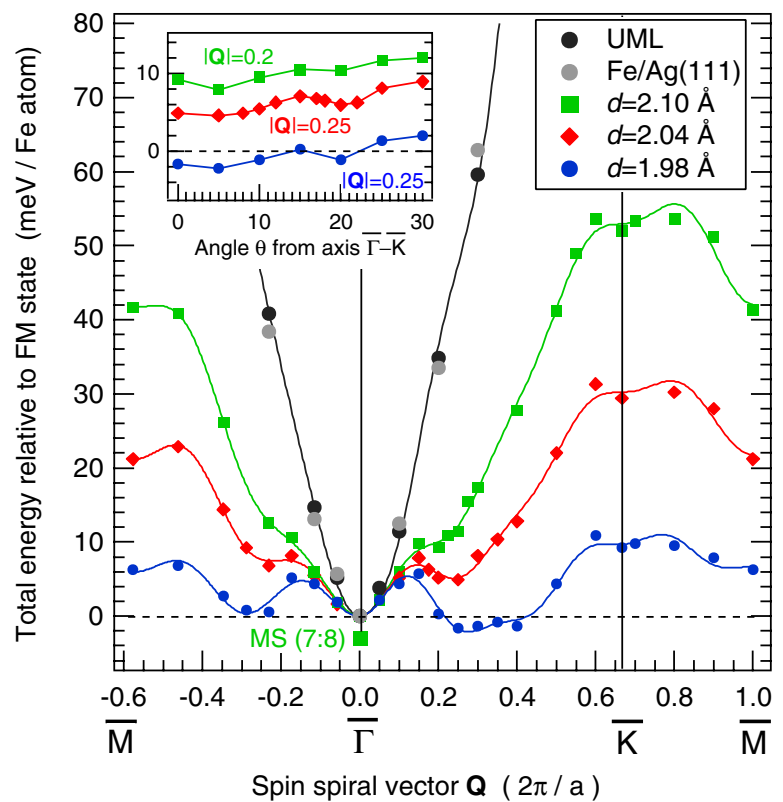

FIG. 4 (color online). Energy $E(\mathbf{Q})$ of spin spirals along high symmetry directions for a hexagonal Fe ML without substrate (UML), on $\operatorname{Ag}(111)$ with $d=2.10 \AA$, and on $\operatorname{Ir}(111)$ at different interlayer spacings $d$. Symbols mark values from first-principles calculations while the lines are a fit to the Heisenberg model including interactions up to eight nearest in-plane neighbors. The inset shows the energy for spin spirals with constant $|\mathbf{Q}|$ in units of $2 \pi / a$ vs the angle $\theta$ describing the path from the $\overline{\Gamma K}\left(\theta=0^{\circ}\right)$ to the $\overline{\Gamma M}$ direction $\left(\theta=30^{\circ}\right)$.

$1.98 \AA$ the plateau first evolves into a local and eventually into a global energy minimum with a slight shift to $|\mathbf{Q}| \approx$ $0.25 \times 2 \pi / a$. As in the collinear calculations, the increasing Fe-Ir hybridization induces an instability of the FM state. A closer inspection of this (local) energy minimum reveals only a weak variation along the circular path around $\bar{\Gamma}$ (see inset of Fig. 4). This gives rise to a centrosymmetric energy valley around $\bar{\Gamma}$ which coincides with the ringlike arrangement of the spots in the FT of the SPSTM image in Fig. 2(e).

Single helical spin spirals, so-called single- $Q$ states, evidently do not represent the experimentally observed magnetic structure but can form the basis for its understanding. Any linear superposition of degenerate single- $Q$ states, which results in a constant spin moment at every atomic site, forms a multiple- $Q$ state and is a degenerate solution within the Heisenberg model. However, particular linear combinations may gain energy on the order of $10 \mathrm{meV} /$ atom [5], due to the presence of higher order exchange interactions such as, e.g., the biquadratic or four-spin interactions. Such terms are beyond the Heisenberg model but implicitly included in the exchange-correlation functional (GGA). The excellent agreement of the experimentally determined size of the magnetic structure in Fourier space, given by $|\mathbf{G}|$, with the radius $|\mathbf{Q}|$ of the ringlike energy plateau found in our calculations, suggests that multiple- $Q$ states can be constructed which reproduce the observed magnetic unit cell. Such states will in general be noncollinear but may be quite similar to the (7:8) mosaic structure, a collinear approximation of the real magnetic structure. Deviations from collinearity seem reasonable since some of the magnetic moments are frustrated in the (7:8) mosaic structure. However, the lack of in-plane contrast in experiment and the good agreement of the calculated local density of states with the measured tunneling spectrum suggest that the true magnetic structure is not much different from the one discussed here. A more detailed understanding of this novel magnetic structure will be a challenge for future investigations. Our work suggests that ultrathin hexagonal magnetic films on substrates of late $4 d$ and $5 d$ transition metals may exhibit a rich field of complex magnetic phases, paid little attention to in the past.

Financial support from the DFG (SFB 668), the Stifterverband für die Deutsche Wissenschaft, and the Interdisciplinary Nanoscience Center of Hamburg is gratefully acknowledged.

*Electronic mail: kbergman@physnet.uni-hamburg.de

[1] S. Heinze et al., Science 288, 1805 (2000).

[2] A. Kubetzka et al., Phys. Rev. Lett. 94, 087204 (2005).

[3] P. Krüger, M. Taguchi, and S. Meza-Aguilar, Phys. Rev. B 61, 15277 (2000).

[4] D. Spišák and J. Hafner, Phys. Rev. B 61, 12728 (2000).

[5] P. Kurz, G. Bihlmayer, K. Hirai, and S. Blügel, Phys. Rev. Lett. 86, 1106 (2001).

[6] S. Heinze et al., Appl. Phys. A 75, 25 (2002).

[7] Y. Tsunoda, J. Phys. Condens. Matter 1, 10427 (1989).

[8] K. Knöpfle, L. M. Sandratskii, and J. Kübler, Phys. Rev. B 62, 5564 (2000).

[9] T. Asada and S. Blügel, Phys. Rev. Lett. 79, 507 (1997).

[10] P. Krüger, Phys. Rev. B 64, 094404 (2001).

[11] D. Qian et al., Phys. Rev. Lett. 87, 227204 (2001).

[12] D. Spišák and J. Hafner, Phys. Rev. B 67, 134434 (2003).

[13] O. Pietzsch et al., Rev. Sci. Instrum. 71, 424 (2000).

[14] C. Busse et al., Phys. Rev. B 68, 201401(R) (2003).

[15] M. Bode, Rep. Prog. Phys. 66, 523 (2003).

[16] http://www.flapw.de.

[17] J. P. Perdew, K. Burke, and M. Ernzerhof, Phys. Rev. Lett. 77, 3865 (1996).

[18] The calculated magnetocrystalline anisotropy energy (MAE) for the FM state is $1.3 \mathrm{meV} / \mathrm{Fe}$ atom and leads to an easy axis perpendicular to the surface. Because of the enormous computational effort, we could not determine the MAE for the (7:8) state; however, we assume that it is similar to the FM state.

[19] D. Wortmann et al., Phys. Rev. Lett. 86, 4132 (2001).

[20] J. Tersoff and D. R. Hamann, Phys. Rev. Lett. 50, 1998 (1983).

[21] V. A. Ukraintsev, Phys. Rev. B 53, 11176 (1996).

[22] Note that the (7:8) MS is not contained in the solutions of this calculation and remains the most favorable magnetic state as indicated in Fig. 4. 